\title{
Implications of Pathogenesis by Erwinia amylovora on Rosaceous Stigmas to Biological Control of Fire Blight
}

\author{
K. B. Johnson, T. L. Sawyer, V. O. Stockwell, and T. N. Temple
}

Department of Botany and Plant Pathology, Oregon State University, Corvallis 97331-2902.

Accepted for publication 8 October 2008.

\begin{abstract}
Johnson, K. B., Sawyer, T. L., Stockwell, V. O., and Temple, T. N. 2009. Implications of pathogenesis by Erwinia amylovora on rosaceous stigmas to biological control of fire blight. Phytopathology 99:128-138.

As a prerequisite to infection of flowers, Erwinia amylovora grows epiphytically on stigmas, which provide a conducive habitat for bacterial growth. Stigmas also support growth of several other bacterial genera, which allows for biological control of fire blight; although, in practice, it is very difficult to exclude E. amylovora completely from this habitat. We investigated the dynamics of growth suppression of E. amylovora by comparing the ability of virulent and avirulent strains of E. amylovora to compete with each other on stigmas of pear, apple, and blackberry, and to compete with a co-inoculated mixture of effective bacterial antagonists. When strains were inoculated individually, virulent $E$. amylovora strain Ea153N attained the highest population size on stigmas, with population sizes that were approximately double those of an avirulent $h r p L$ mutant of

greater extent than the virulent strain. Unexpectedly, the virulent strain enhanced the population size of the antagonist mixture. Similarly, a small dose of virulent Ea153N added to inoculum of an avirulent $h r p L$ mutant of Ea153 significantly increased the population size of the avirulent strain. A pathogenesis-gene reporter strain, Ea153 $d s p E:: g f p$, was applied to flowers and a subset of the population expressed the green fluorescent protein while growing epiphytically on stigmas of apple. These results are consistent with the hypothesis that virulent E. amylovora modifies the epiphytic habitat presented by the stigma through a pathogenesis-related process, which increases host resources available to itself and, coincidentally, to nonpathogenic competitors. Over nine orchard trials, avirulent Ea153 hrpL significantly suppressed the incidence of fire blight four times compared with six for the antagonist mixture. The degree of biological control achievable with an avirulent strain of $E$. amylovora likely is limited by its inability to utilize the stigmatic habitat to the same degree as a virulent strain.
\end{abstract} Ea153 or the bacterial antagonists. In competition experiments, growth of the avirulent derivative was suppressed by the antagonist mixture to a

Infection of flowers by Erwinia amylovora is an important phase in the development of fire blight of pear and apple (15, $28,34)$. In orchards, floral infection is preceded by an epiphytic phase, where the pathogen grows on the surfaces of the stigmas (33). On rosaceous plants, this surface represents a nutrient-rich, hydrated habitat on which epiphytic pathogen populations can grow to exceed $10^{7} \mathrm{CFU}$ per flower (which represents to $10^{9}$ to $10^{10} \mathrm{CFU} / \mathrm{g}$ of stigmatic tissue) (15). Large epiphytic populations on stigmas greatly increase the likelihood of pathogen cells sliding down the surface of the style into the nectary $(25,33)$, where infection occurs, and also facilitate insect-mediated dispersal to other flowers $(15,34)$.

The stigmatic habitat of rosaceous plants is apparently nonselective $(14,15,17,28)$ and, consequently, it supports growth of other commensal epiphytes (bacteria and yeasts), some of which are antagonistic to E. amylovora $(16,26,29)$. Once established on stigmas, antagonistic bacteria utilize the mechanisms of competitive exclusion (18) and antibiosis (29) to suppress epiphytic growth of the pathogen. In field studies designed to evaluate biological control of fire blight, antagonists are sprayed at high doses onto flowers followed, after several days, by a lower-dose pathogen inoculation. With this protocol, antagonists typically reduce infection by 40 to $60 \%(16,29)$. Epiphytic establishment and growth of E. amylovora on flowers also is suppressed only partially, even when the environment favors rapid growth and dis-

Corresponding author: K. B. Johnson;

E-mail address: johnsonk@science.oregonstate.edu

doi:10.1094/PHYTO-99-2-0128

(C) 2009 The American Phytopathological Society
Additional key words: Pantoea agglomerans, Pseudomonas fluorescens. persal of antagonists. Why antagonist treatments, when given advantages of initial dose and time, achieve only partial suppression of pathogen activity on flowers is not well understood.

Several studies concerned with ecology of bacterial epiphytes in the phyllosphere have shown that pathogens can be better adapted than nonpathogens for epiphytic growth and survival on healthy plant surfaces. For example, compared with nonpathogens, pathogens were found to possess a greater ability to access and survive in "protected sites" on bean leaf surfaces (39). Similarly, after field inoculation, the epiphytic population sizes of avirulent $h r p$ mutants of bacterial plant pathogens on surfaces of bean (9), tomato (6), and pepper (38) were significantly smaller than those of the parental, pathogenic strain, suggesting that the loss of virulence contributed to a reduction in epiphytic fitness. In contrast, a pathogenic strain and a derived hrp mutant had similar growth rates and epiphytic population sizes in the nutrient-rich spermospheres of preemergent bean seedlings (9).

Avirulent strains of E. amylovora have been evaluated as agents for suppression of fire blight in several studies $(5,7,32,41)$. Most recently, avirulent $h r p$ regulatory mutants were shown to partially protect greenhouse-grown apple seedlings from shoot and blossom infection by the pathogenic, parental strain $(5,32)$. Differences in epiphytic fitness among virulent and avirulent strains, however, were not evaluated in these efforts, nor were avirulent strains of E. amylovora evaluated for their ability to protect flowers under field conditions. The purpose of this study was to develop insights into the competitive ability of E. amylovora as an epiphyte on surfaces of rosaceous flowers, and to relate this ability to the current understanding of fire blight biocontrol. Our approach utilized de Wit replacement series experiments $(8,40)$ to evaluate competitive interactions among a pathogenic strain of $E$. 
amylovora, an avirulent hrpL mutant of this strain, and an effective antagonist mixture consisting of a strain of Pantoea agglomerans mixed with a strain of Pseudomonas fluorescens. Analysis of competition experiments led to additional experiments concerned with expression of a pathogenesis-related gene in epiphytic populations of virulent E. amylovora. We also addressed the degree to which an avirulent hrpL mutant of E. amylovora may protect flowers from fire blight in an orchard environment.

\section{MATERIALS AND METHODS}

Bacterial strains. The strains described below were used in growth-chamber, screenhouse, and field experiments. E. amylovora strain Ea153N is a spontaneous, nalidixic-acid-resistant $(100 \mu \mathrm{g} / \mathrm{ml})$ selection of Ea153, which was originally isolated from a diseased apple. Ea153N shows virulence characteristics typical of other North American strains of this pathogen and has been used as a representative strain in numerous field studies $(14,16,17,27,29,31)$. An avirulent $h r p L:: T n 10$ miniKan insertion mutant of Ea153 (Ea153 hrpL with resistance to kanamycin $(50 \mu \mathrm{g} / \mathrm{ml})$ was constructed using allelic-exchange mutagenesis with pCPP1032 provided by S. Beer (Cornell University, Ithaca, NY) (36); epiphytic survival of this strain in an orchard environment was investigated previously (31). Virulent reporter strain Ea153 $d s p E:: g f p$ was created by fusing the $h r p L$-regulated promoter of $d s p E$ of $E$. amylovora $(2,3)$ to a promoterless gene for the green fluorescent protein (GFP), gfp3. The $d s p E$ promoter was amplified from Ea153 with primers based on those designed by Zhao et al. (42). The amplicon was ligated into pDFI 125.1, a broad-host-range plasmid with promoterless $g f p 3$ and resistance to kanamycin, which was provided by J. Chang (Oregon State

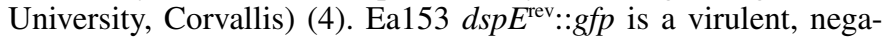
tive control for the reporter construct; it was constructed with the $d s p E$ promoter in reverse orientation to promoterless $g f p 3$. The sequence and orientation of the $d s p E$ promoter for both constructs was confirmed by sequence analysis. Ea153 $g f p$ is a positive control for $g f p 3$ expression; it carries pDFI 124.B1 with $g f p 3$ regulated by a constitutive promoter (4). Each plasmid was moved into Ea153 by electroporation (21); virulence of each construct of Ea153 was confirmed in an immature pear fruit assay.

Pantoea agglomerans strain $\mathrm{PaC} 9-1 \mathrm{~S}$ is a spontaneous streptomycin- and rifampicin-resistant (each $100 \mu \mathrm{g} / \mathrm{ml}$ ) selection of PaC9-1, with characteristics similar to the original strain (13); this strain is an excellent colonist of pear and apple flowers (17, 28,31), and has been commercialized to suppress floral infection by E. amylovora (BlightBan C9-1; NuFarm Americas, Burr Ridge, IL). Pseudomonas fluorescens strain A506 $\Delta a p r X$ (PfA506 $\triangle a p r X)$ is an extracellular protease-deficient derivative of PfA506 generated by allelic-exchange mutagenesis resulting in an $\approx 300$-bp deletion in the gene aprX, which encodes for an extracellular metalloprotease (1). The original strain, PfA506 (18), is resistant to streptomycin $(100 \mu \mathrm{g} / \mathrm{ml})$ and rifampicin $(100 \mu \mathrm{g} / \mathrm{ml})$ and has been commercialized as an agent to suppress fire blight via competitive exclusion (BlightBan A506; NuFarm Americas). Loss of the protease by PfA506 $\triangle a p r X$ results in a phenotype that is unable to inactivate a peptide antibiotic produced by many strains of Pantoea agglomerans; consequently, a strain mixture of PfA506 $\triangle a p r X$ and PaC9-1S has shown enhanced fire blight suppression (26).

For most experiments, initial inoculum of the strains consisted of lyophilized cells prepared at a titer of $1 \times 10^{11}$ to $1 \times 10^{12}$ CFU/g (27). Lyophilized preparations were produced once and stored at $-70^{\circ} \mathrm{C}$, with subsamples withdrawn for use in experiments.

Competition experiments in a growth chamber. Four replacement series experiments $(8,38)$ were conducted in growth chambers to investigate competitive interactions between epiphytic populations of virulent Ea153N, avirulent Ea153 hrpL, PfA506 $\triangle a p r X$, and $\mathrm{PaC} 9-1 \mathrm{~S}$ on stigmas of rosaceous flowers. Each experiment was designed with strain component 1 and strain component 2 in the following ratios: 1.0:0.0, 0.9:0.1, 0.5:0.5, 0.1:0.9, and 0.0:1.0. Strain component pairings were: (i) PfA506 $\triangle a p r X$ with PaC9-1S, (ii) Ea153N with Ea153 hrpL, (iii) Ea153N with a 0.5:0.5 mix of PfA506 $\triangle a p r X$ and PaC9-1S composing strain component 2, and (iv) Ea153 hrpL with a 0.5:0.5 mix of PfA506 $\triangle a p r X$ and PaC9-1S composing strain component 2. The four experiments were first performed with flowers of pear (Pyrus communis cv. Bartlett), followed by apple (Malus pumila cv. Gala) and then blackberry (Rubus armeniacus naturalized 'Himalayan Giant'). Experiments were arranged as randomized block design with four replications of each plant species blocked by chamber and by time. Within each replication, the experimental unit comprised eight flowers that received the same strain and ratio combination. Individual replications for pear were inoculated between 25 March and 5 April 2005; for apple, between 13 and 27 April; and for blackberry, between 23 May and 5 June.

Flowers for the experiments were obtained from field-grown trees located at the Oregon State University, Department of Botany and Plant Pathology Field Laboratory near Corvallis or, in the case of blackberry, from nearby naturalized hedges. Blossombearing branches were pruned from trees 4 to 5 days before first bloom and stored at $5^{\circ} \mathrm{C}$ with the basal ends submerged in water. At the start of an experimental replication, branches were incubated in the laboratory $\left(20\right.$ to $\left.23^{\circ} \mathrm{C}\right)$, where flowers opened within 1 to 2 days. Following protocols of Pusey (24), new flowers (cupped petals and nondehiscent anthers) were harvested with peduncle intact $(1.5 \mathrm{~cm}$ in length) and placed onto 80 -well, polypropylene microcentrifuge tube racks (Fisher Scientific, Pittsburgh) with the basal ends of the peduncles submerged in sterile $10 \%$ sucrose. Suspensions of Ea153N, Ea153 hrpL PfA506 $\Delta a p r X$, and $\mathrm{PaC}$-1S were prepared by resuspending freeze-dried preparations in sterile distilled water to achieve a concentration of $1 \times$ $10^{7} \mathrm{CFU} / \mathrm{ml}$; the suspensions were mixed to obtain the appropriate series of inoculum ratios. Each flower was inoculated by pipette with $10 \mu \mathrm{l}$ of a prepared inoculum suspension onto the stigmas. Racks holding inoculated flowers were transferred to 10-liter plastic, lidded boxes (Rubbermaid, Wooster, $\mathrm{OH}$ ), to which 1 liter of a $40 \%$ glycerol solution ( $\mathrm{vol} / \mathrm{vol}$ ) was added to maintain a relative humidly of 93 to $94 \%$ in closed boxes (24). Boxes containing the racks of flowers were incubated in a nonlighted growth chamber (VWR Scientific Model 2105; Sheldon Mfg., Inc., Cornelius, OR) set to $16^{\circ} \mathrm{C}$.

Population sizes of the bacterial strains on individual flowers were determined by dilution plating. Eight flowers (an experimental unit) were sampled at 0 and at $96 \mathrm{~h}$ after inoculation, an interval that we have determined previously to be useful for evaluating epiphytic growth on stigmas (17). Pistils from each flower were excised and placed individually in test tubes containing $1 \mathrm{ml}$ of sterile potassium phosphate buffer $(0.04 \mathrm{M}, \mathrm{pH} 6.5)$. Test tubes containing pistils were sonicated for $2 \mathrm{~min}$ and vortexed to dislodge the bacteria from the stigmatic surfaces. After sonication, $10 \mu \mathrm{l}$ of the sample buffer and two 100-fold dilutions were spread onto selective culture media $(17,29)$. Pseudomonas agar $\mathrm{F}$ (PAF; Difco Laboratories, Detroit) with cycloheximide at $50 \mu \mathrm{g} / \mathrm{ml}$ and rifampicin at $50 \mu \mathrm{g} / \mathrm{ml}$ was used for selective recovery of PfA506 $\triangle a p r X$ and PaC9-1S; these strains were counted on the same plate after 3 to 4 days of incubation and were differentiated based on colony size, color, and morphology. CCT medium (12) or PAF medium containing nalidixic acid at $50 \mu \mathrm{g} / \mathrm{ml}$ was used for recovery of Ea153N; CCT or PAF and Miller Schroth medium (22) containing sorbitol and amended with kanamycin at $50 \mu \mathrm{g} / \mathrm{ml}$ was used for selective recovery of Ea153 $\mathrm{hrpL}$.

Competition experiments in a screenhouse. In spring 2004, a replacement series experiment was conducted under fluctuating temperature conditions on pear and apple trees growing in an unheated, 15.5-by-60-m screenhouse located at the Oregon State 
University, Department of Botany and Plant Pathology Field Laboratory near Corvallis. Trees in the screenhouse ranged from 5 to 9 years old and were protected from rain by a translucent, fiberglass roof, and from insect visitations by 2-by-2-mm steel screen walls. The experiment was designed with strain component 1 and strain component 2 in the following ratios: 1.0:0.0, 0.5:0.5, and $0.0: 1.0$. Strain component pairings were (i) Ea153N with a $0.5: 0.5$ mix of PfA506 $\triangle a p r X$ and PaC9-1S composing strain component 2 and (ii) Ea $h r p L$ with a 0.5:0.5 mix of Pf506 $\triangle a p r X$ and PaC9-1S composing strain component 2 . The experiment was arranged as a randomized block design with a total of five replications; two replications were conducted with pear cv. Bartlett, one with crab apple cv. Snowdrift, and two with apple cv. Golden Delicious. Inoculation dates of individual replications were 26 March and 1 April for pear, 5 April for crab apple, and 7 and 12 April for Golden Delicious apple. An additional treatment was created by enclosing three of the six experimental trees in chambers 1.7 by 1.7 by $2 \mathrm{~m}$ (height) in size, and heating these chambers with two 100-watt light bulbs during a 96-h incubation period; this treatment increased the postinoculation temperature the trees experienced by $\approx 4^{\circ} \mathrm{C}$ (17). The chambers were constructed with frames made of polyvinyl chloride pipe, and with walls and roof composed of clear, polyethylene sheeting. The polyethylene sheeting was hung on the frames immediately after the inoculation treatments. All replications of the inoculation/incubation protocol were initiated in the morning between 8:00 and 10:00 a.m.

To initiate an experimental replication, individual branches on each of six trees (three enclosed and three nonenclosed) were randomly assigned a bacterial strain treatment. On each branch, eight newly opened flowers (nondehiscent anthers) were marked by securing color-coded tape around the peduncle. Suspensions of bacterial strains $\left(1 \times 10^{7} \mathrm{CFU} / \mathrm{ml}\right)$ were prepared as described above and misted onto flowers to near runoff with hand-held, adjustable trigger sprayers (0.2-liter capacity). For each strain combination, 24 marked flowers (4 from each of the enclosed trees and 4 from each of the nonenclosed trees) were sampled 30 min after inoculation; the other 24 flowers were sampled after $96 \mathrm{~h}$ of incubation. In the laboratory, the population sizes of inoculated strains on individual flowers were estimated as described above. Ambient screenhouse and enclosure temperatures were monitored and recorded with electronic temperature sensors (Hobo U10; Onset Computer Corp., Pocasset, MA) set to hourly sampling intervals.

Epiphytic yields of virulent and avirulent $\boldsymbol{E}$. amylovora. In spring 2006 and 2007, epiphytic yield (i.e., the population size after a designated time period) experiments were conducted with strains Ea153N and Ea153 hrpL inoculated onto flowers of pear and apple. In addition to each strain alone, one mixture treatment was prepared to examine the effect of a small number of virulent cells in the initial inoculum on growth of the avirulent strain. For all treatments, the total concentration of inoculum applied to flowers was $1 \times 10^{7} \mathrm{CFU} / \mathrm{ml}$. The ratio of virulent to avirulent $\mathrm{CFU}$ in the inoculum of the mixture treatment, which was assayed after mixing, ranged from 1:11 to 1:21, depending on the experiment. The experiment was conducted in a growth chamber with detached pear (cv. Bartlett, 2007) and apple (cv. Gala, 2006) flowers, and twice in the screenhouse in 2007 on chambered trees (see above) of Bartlett pear and Golden Delicious apple. Both growth-chamber and screenhouse experiments were designed as randomized block designs with four replications, three treatments, and 16 flowers composing an experimental unit. The inoculation, incubation, and processing protocols for flowers in each experiment were as described above. The incubation period from inoculation to final sampling was $96 \mathrm{~h}$.

$\boldsymbol{d} \boldsymbol{s p} \boldsymbol{E}$ promoter expression in epiphytic $\boldsymbol{E}$. amylovora. We detected differences in the population dynamics of Ea153N and the $h r p L$ mutant on flowers. The following experiment was devised to determine whether pathogenicity-related genes are expressed by the pathogen on stigmas. We chose the pathogenesis- related gene $d s p E$ as an indicator of expression of pathogenesis because this gene was highly expressed by E. amylovora during early stages of infection of immature pear fruit $(2,3,42)$. Detached apple flowers (cv. Gala) were inoculated with virulent reporter strains of E. amylovora to monitor the temporal expression of the pathogenesis-related gene, $d s p E$, in epiphytic populations sampled from floral stigmas. For each experimental replication, strains Ea153 $d s p E:: g f p$, Ea153 $d s p E^{\mathrm{rev}}:: g f p$, and Ea153 $g f p$ were each inoculated onto 92 newly open flowers prepared and placed into microcentrifuge tube racks as described above. Inoculum of each strain was obtained from cultures grown overnight in LuriaBertani broth, a medium in which $g f p$ is not expressed in Ea153 $d s p E:: g f p$. Prior to inoculation, cells were centrifuged and washed twice, and resuspended in sterile distilled water to achieve a concentration of $1 \times 10^{7} \mathrm{CFU} / \mathrm{ml}$. Each flower was inoculated by pipette with $10 \mu \mathrm{l}$ of a prepared inoculum suspension onto the stigmas. At the same time, vials containing $1 \mathrm{ml}$ of modified minimal salts medium (11) were inoculated with each construct as additional controls. In this medium, $g f p$ is expressed in Ea153 $d s p E:: g f p$ and Ea153 $g f p$ but not in the negative control Ea153

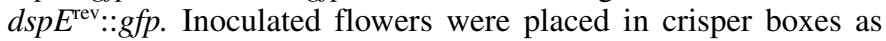
described above and the inoculated vials of minimal media were placed on a rotary shaker; both flowers and vials were incubated in a growth chamber at $16^{\circ} \mathrm{C}$.

At $0,12,24,36,48,60,72,84$, and $96 \mathrm{~h}$ after inoculation, the pistils from six flowers inoculated with E. amylovora 153 $d s p E:: g f p$ and from three flowers inoculated with Ea153 $d s p E^{\text {rev }}:: g f p$ or Ea153 $g f p$ were sampled and washed individually in phosphate buffer as described above. Dilutions of the wash from three flowers inoculated with Ea153 $d s p E:: g f p$ were spread onto nutrient agar to obtain an estimate of epiphytic population size. Bacterial cells were prepared for microscopic examination by staining with 4 '-6-diamidino-2-phenylindole (DAPI) $(3 \mu \mathrm{M})$ for $20 \mathrm{~min}$, followed by centrifugation, resuspension, and fixation in $2 \%$ paraformaldehyde, and centrifugation and resuspension in $50 \%$ glycerol for viewing. Also at each sampling time, cells in minimal salts medium were centrifuged and resuspended in phosphate buffer prior to staining. Prior to fixation, construct cultures in minimal salts media were dilution plated in triplicate onto nutrient agar to obtain an estimate of population size. Stained cells were placed onto glass slides and viewed at $\times 400$ on a Leica DMRB epifluorescent microscope equipped with a $50-\mathrm{W} \mathrm{Hg}$ lamp. A Chroma 41017 filter set and a Leica filter cube A were used for visualizing GFP and DAPI stain, respectively. The incidence of expression of GFP was scored in a subsample of 100 cells from each flower or media vial; the experiment was repeated four times.

Fire blight suppression by avirulent $\boldsymbol{E}$. amylovora. Field experiments evaluating suppression of fire blight by Ea153 hrpL were conducted from 2004 to 2007 in 25- to 47-year-old, 0.4- to 0.5-ha blocks of pear (cvs. Bartlett and Bosc) and apple (cvs. Golden Delicious and Rome Beauty) located at the Oregon State University, Botany and Plant Pathology Field Laboratory near Corvallis. Additional treatments in the experiments included a 1:1 mixture of PfA506 $\triangle a p r X$ and PaC9-1S and a 1:1:1 mixture of PfA506 $\Delta a p r X$, PaC9-1S, and Ea153 hrpL. At the time of treatment, freeze-dried bacterial preparations were resuspended in water (local groundwater sources) to achieve a total concentration of $1 \times 10^{8} \mathrm{CFU} / \mathrm{ml}$. Trees treated with water or the antibiotics streptomycin sulfate (Agristrep 17\%, $100 \mu \mathrm{g} / \mathrm{ml}$ a.i.; Nufarm Americas Inc.) and oxytetracycline (Mycoshield, $200 \mu \mathrm{g} / \mathrm{ml}$ a.i.; Nufarm Americas Inc.) were included as comparative controls. Treatments were assigned to individual trees in a randomized complete block design with four or five replications. Relative bloom density and location in the orchard were both considered in the assignment of trees to individual blocks.

Treatments were applied to trees in the early morning between 6:30 and 8:00 a.m. The bacteria were applied at 30 to $40 \%$ bloom 
and again at 70 to $80 \%$ bloom. Water and antibiotic treatments were applied at 70 to $85 \%$ bloom and repeated 3 to 6 days later during full bloom. All treatments were applied to near run-off using 12-liter backpack sprayers fitted with adjustable nozzles attached to a hand-held spray wand. Approximately 3 to 4 liters of a treatment suspension was applied to a tree on each date. Near full bloom, resuspended freeze-dried inoculum $\left(5 \times 10^{5}\right.$ to $1 \times$ $10^{6} \mathrm{CFU} / \mathrm{ml}$ ) of virulent Ea153N was misted $(\approx 2$ liters per tree) onto flowers to ensure adequate disease.

Population sizes of PfA506 $\Delta a p r X$, PaC9-1S, Ea153 hrpL, and Ea153N were monitored on each tree that received a bacterial or water treatment by sampling eight flowers from each tree near petal fall (5 to 8 days after the pathogen inoculation). Sampled flowers were placed into wells of a microtiter dish, transported to the lab, and individually washed and dilution plated as described above. Counts of fire blight infections were made weekly, beginning when symptoms became apparent and continuing for 3 to 4 weeks. Diseased blossom clusters observed and pruned from each tree were summed over all observation dates. Prior to bloom, the total number of blossom clusters on each tree was estimated by visual inspection.

Data analysis. In all experiments, the estimated population size of an inoculated strain on an individual flower was $\log _{10}$-transformed, then averaged with estimates from the other flowers within an experimental unit to create a replicate mean for each inoculum proportion or treatment. Replicate means of $\log _{10}$-transformed population size were subjected to analysis of variance (ANOVA) (PROC ANOVA; Statistical Analysis Systems, Cary, NC). Similarly, to display the data graphically, replicate means of strain population size were computed from nontransformed data, from which overall means and standard errors were plotted against inoculum proportion $(8,40)$.

ANOVA of the replacement series data from the growth chamber and screenhouse experiments were analyzed following procedures outlined by Wilson and Lindow (40). The null hypothesis, that the outcome of intercomponent competition equals that of intracomponent competition (i.e., the strains compete equally with each other), was tested by performing ANOVA on the mean $\log _{10}$ population size of a strain component at each inoculum proportion according to the following model: $\log _{10}$ (population size ij $_{i j}$ ) $\log _{10}\left(\right.$ inoculum $\left._{\text {proportion }}\right)=$ mean $_{i}+$ normal error $_{i j}$, where $i$ is the inoculum proportion and $j$ is the replicate. This model assumes that population size is log normally distributed, which is generally true of epiphytic bacterial populations $(10,19)$. In this model, if the null hypothesis is correct, each inoculum proportion should result in a similar estimate of the true mean. If the null hypothesis is rejected, evaluation of the plotted data can determine if a strain component benefits from or is antagonized by the other strain component. The model was run independently for each strain component in an experiment.

For the field experiments, the total number of diseased blossom clusters per treated tree was converted first to a disease incidence by dividing by the estimated number of blossom clusters on each tree, and then converted to a relative disease incidence by dividing thorough by the mean disease incidence on the water-treated trees. Relative disease incidence values data were subjected to ANOVA; Fisher's protected least significant difference test at $P=$ 0.05 was used for separation of treatment means.

\section{RESULTS}

Competition experiments in growth chambers. Four replacement series experiments were conducted with detached flowers of pear, apple, and blackberry incubated for $96 \mathrm{~h}$ at $16^{\circ} \mathrm{C}$. The experiment with PfA506 $\Delta a p r X$ paired against $\mathrm{PaC} 9-1 \mathrm{~S}$ resulted in a typical "equal competitor" pattern where the final epiphytic yields (or resultant population size) of each strain showed a pattern consistent with the initial inoculum dose (i.e., the yields of each strain in mixture with the other strain maintained an approximately linear relationship with the initial inoculum proportion) (Fig. 1).

Replacement series experiments that involved strains of $E$. amylovora deviated from the equal competitor pattern, although each strain pairing resulted in generally similar patterns among the three hosts (Fig. 2). Virulent Ea153N, when inoculated alone, had epiphytic yields that averaged two to three times more CFU than solitary inoculations of the nonpathogenic strains (i.e., PfA506 $\triangle a p r X$, PaC9-1S, and Ea153 hrpL). The 0.5:0.5 inoculum mixture of PfA506 $\triangle a p r X$ and PaC9-1S, which composed strain component 2, strongly antagonized both Ea153N and Ea153 hrpL on all three host flowers; although population sizes of Ea153N in mixture with the antagonist strains in any proportion were greater than the corresponding population sizes of Ea153 hrpL (compare Fig. 2A, D, and $\mathrm{G}$ to $\mathrm{C}, \mathrm{F}$, and $\mathrm{I}$ ). In the experiments with Ea153N, the antagonist strain mixture showed positive deviations from the expectation of linearity with initial inoculum proportion, particularly at an inoculum ratio of $0.9: 0.1 \mathrm{Ea} 153 \mathrm{~N}$ to the antagonist strain component, respectively (Fig. 2A, D, and G). Similarly, Ea153 hrpL, when inoculated in mixture with virulent Ea153N, also showed apparently positive deviations from the expectation of linearity with initial inoculum proportion (Fig. 2B, $\mathrm{E}$, and $\mathrm{H})$, whereas the final population sizes of Ea153N were consistent with the expectation of linearity with initial inoculum proportion.

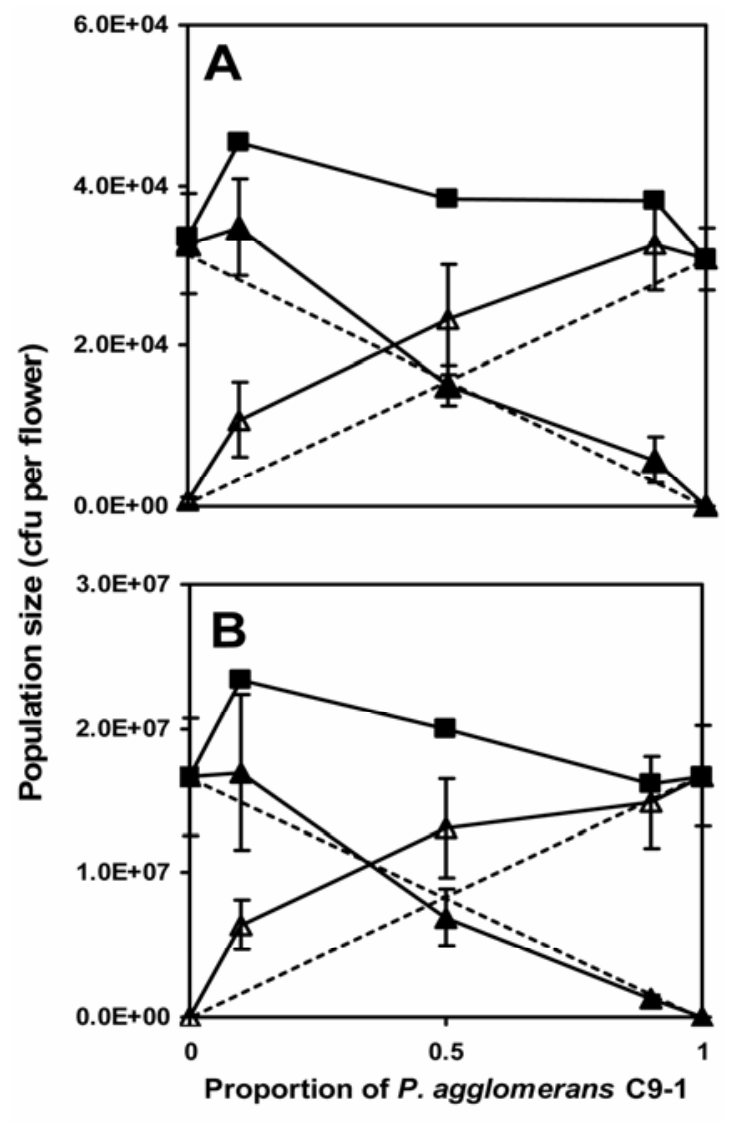

1- proportion $P$. fluorescens A506 $\triangle$ aprX

Fig. 1. Replacement series experiment showing epiphytic population sizes of Pseudomonas fluorescens strain A506 $\Delta a p r X$ (solid triangle) and Pantoea agglomerans strain C9-1S (open triangle) on pear, apple, and blackberry flowers (i.e., overall means from all experiments) at A, 0 and $\mathbf{B}, 96 \mathrm{~h}$ after co-inoculation in varying proportions. Points shown as solid squares represent the sum of the population size of the two strains. Dashed lines represent the expected population size of each strain and the sum of two strains under the null hypothesis that interspecific competition is equivalent to intraspecific competition (39). Bars represent \pm one standard error of the mean. 
ANOVA of epiphytic yields from replacement series experiments that involved strains of E. amylovora confirmed differential competition among paired strain components (Table 1). Both Ea153N and Ea153 hrpL were significantly antagonized ( $P$ values ranged from 0.002 to $<0.0001$ ) by the mixture of PfA506 $\triangle a p r X$ and PaC9-1S. In contrast, on each host, the epiphytic yield of PfA506 $\triangle a p r X$ and $\mathrm{PaC}$-1S significantly benefited ( $P$ values ranged from 0.0023 to 0.0002 ) from competition with Ea153N but not from competition with Ea153 hrpL. The avirulent strain Ea153 hrpL significantly benefited $(P=0.042)$ from the presence of Ea153N on pear flowers but the corresponding positive deviations observed for the avirulent strain on apple and blackberry flowers were not significant (Table 1).

Competition experiments in a screenhouse. The screenhouse replacement series experiment was summarized and analyzed by incubation environment (ambient and chambered) because it is a more significant source of variation in epiphytic population size than is host cultivar (17). Consequently, each mean comprised two inoculations (blocks) of pear, one inoculation of crab apple, and two inoculations of apple. Over the experimental period (29
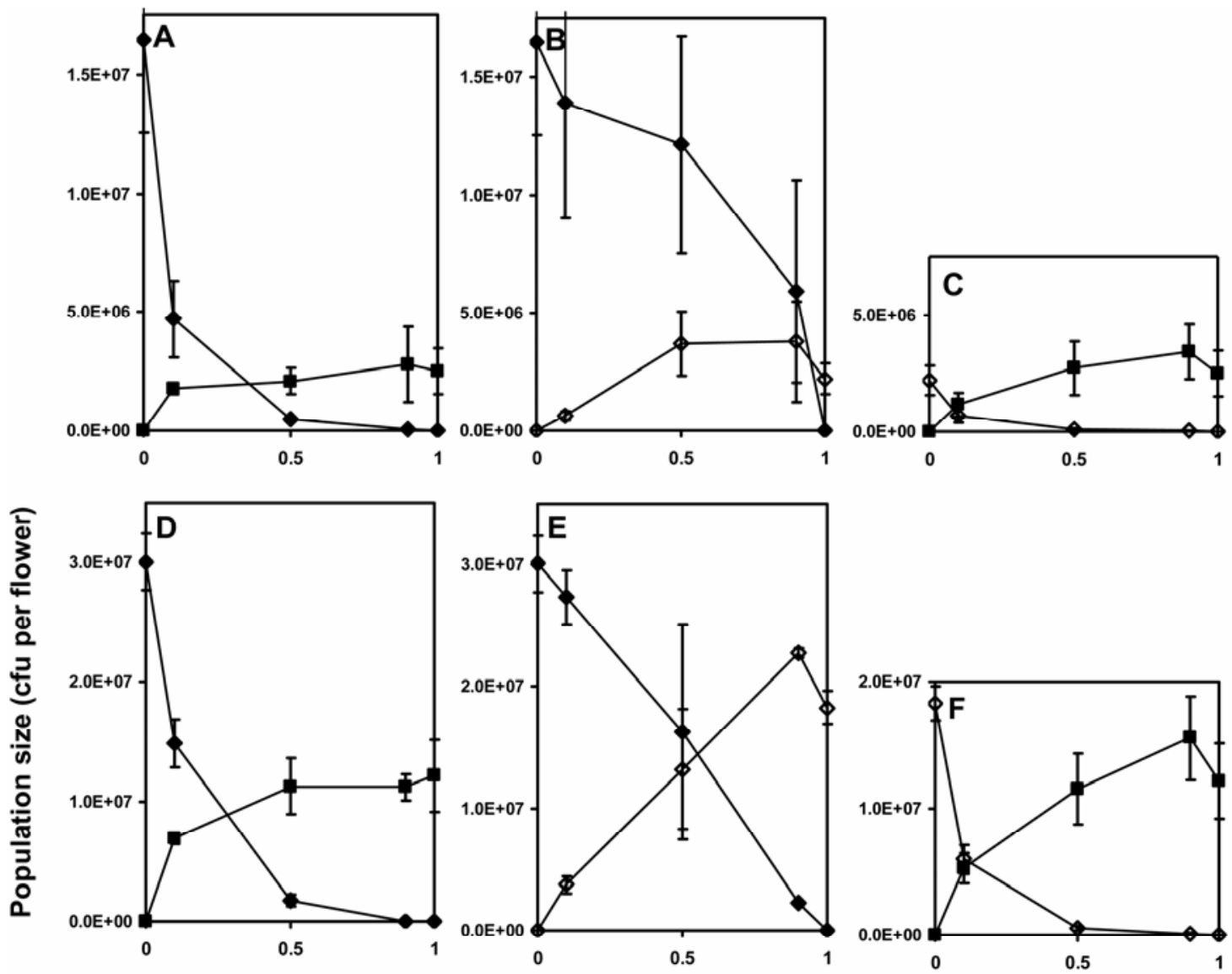

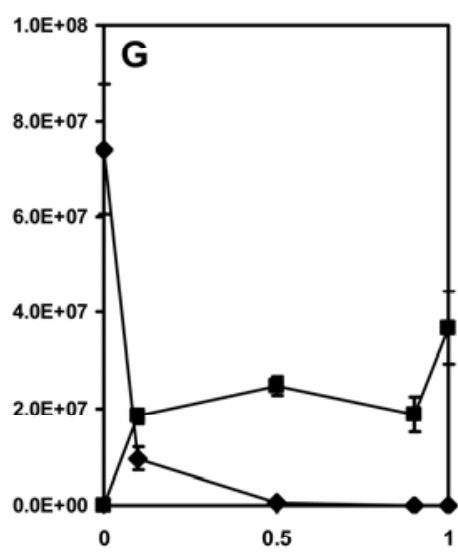

Proportion of PfA506 $\triangle a p r X \&$ Pa C9-1S

1 - proportion of Ea 153N

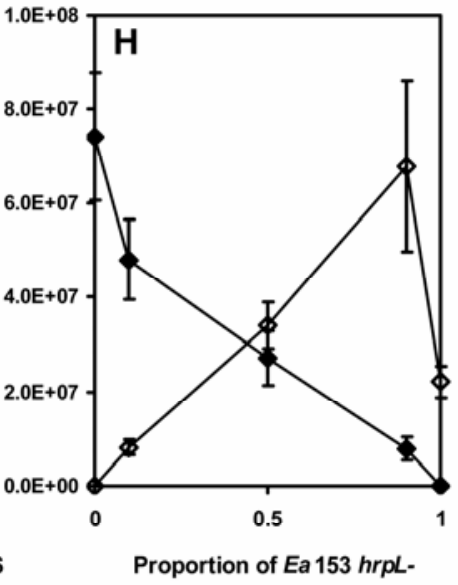

1 - proportion of Ea 153N

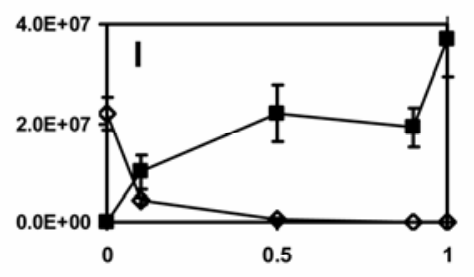

Proportion of PfA506 $\triangle$ aprX \& Pa C9-1S

1 - proportion of Ea $153 \mathrm{hrpl}$ -

Fig. 2. Replacement series experiments showing epiphytic population sizes of A, D, and G, virulent Erwinia amylovora strain $153 \mathrm{~N}$ (solid diamond) paired against a 1:1 mixture of Pseudomonas fluorescens strain A506 $\triangle a p r X$ and Pantoea agglomerans strain C9-1S (solid square); B, E, and H, virulent E. amylovora strain $153 \mathrm{~N}$ (solid diamond) paired against avirulent E. amylovora strain $153 \mathrm{hrpL}$ (open diamond); and $\mathbf{C}$, $\mathbf{F}$, and I, avirulent E. amylovora strain $153 \mathrm{hrpL}$ (open diamond) paired against a 1:1 mixture of Pseudomonas fluorescens strain A506 $\triangle a p r X$ and Pantoea agglomerans strain C9-1S (solid square) on A to C, detached pear; $\mathbf{D}$ to $\mathbf{F}$, apple; and $\mathbf{G}$ to $\mathbf{I}$, blackberry flowers after $96 \mathrm{~h}$ of incubation at $16^{\circ} \mathrm{C}$. In a replacement series experiment, the proportion of each strain component in the initial inoculum is varied (x-axis) while keeping the total inoculum concentration constant. Bars represent \pm one standard error of the mean. 
March to 16 April), the ambient temperature averaged $11^{\circ} \mathrm{C}$ whereas the chambers averaged $15^{\circ} \mathrm{C}$. Pairings of strains of $E$. amylovora with the 0.5:0.5 mixture of PfA506 $\triangle a p r X$ and PaC9$1 \mathrm{~S}$ were similar to the growth-chamber results in that epiphytic yields of the antagonist strain component deviated from the pattern of equal competitors (Fig. 3). In both environments, the measured total population size of PfA506 $\triangle a p r X$ and PaC9-1S in mixture with Ea153N was apparently greater than the expectation of linearity with initial inoculum proportion (Fig. 3), a result that was confirmed by ANOVA (Table 2). Also in the screenhouse, strains of E. amylovora were not antagonized by PfA506 $\Delta a p r X$ and $\mathrm{PaC}$-1S to the same degree as in the growth-chamber experiments although, in the chambered screenhouse environment, the population size of Ea153 hrpL in mixture with the antagonist strains was significantly less $(P=0.0041)$ than the expectation of linearity with initial inoculum proportion (Table 2).

Epiphytic yields of virulent and avirulent $E$. amylovora. In each of the four experiments (two growth chamber and two screenhouse), virulent Ea153N, inoculated by itself onto pear or apple stigmas and incubated for $96 \mathrm{~h}$, resulted in a mean population size that was 2 to 20 times greater than the corresponding size of isolated populations of Ea153 hrpL (Fig. 4); these differences between the mean final population size of isolated populations of Ea153N and Ea153 hrpL were significant in each experiment $(P$ values ranged from 0.02 to $>0.0001)$. Similarly, Ea153 hrpL with a small amount of Ea153N in the initial inoculum obtained a mean population size on pear or apple stigmas that was two to eight times greater than the corresponding size of solitary populations of Ea153 hrpL; these differences between the mean final population size of Ea153 hrpL inoculated in mixture with Ea153N and Ea153 hrpL inoculated by itself also were significant in each experiment ( $P$ values ranged from 0.05 to 0.001 ). For three of the four experiments, the mean final population size of Ea153 hrpL inoculated in mixture with Ea153N was statistically similar to the final population size of virulent Ea153N (Fig. 4B to D); the exception was the growth chamber with detached pear flowers (Fig. $4 \mathrm{~A})$. Also, after $96 \mathrm{~h}$ of incubation, the proportion of Ea153N in the mixed strain treatment increased to a range of 8.0 to $20.3 \%$ compared with a range of 4.7 to $9.1 \%$ in the initial inoculum, showing that, in the mixture, the virulent parental strain multiplied more rapidly than the avirulent derivative.

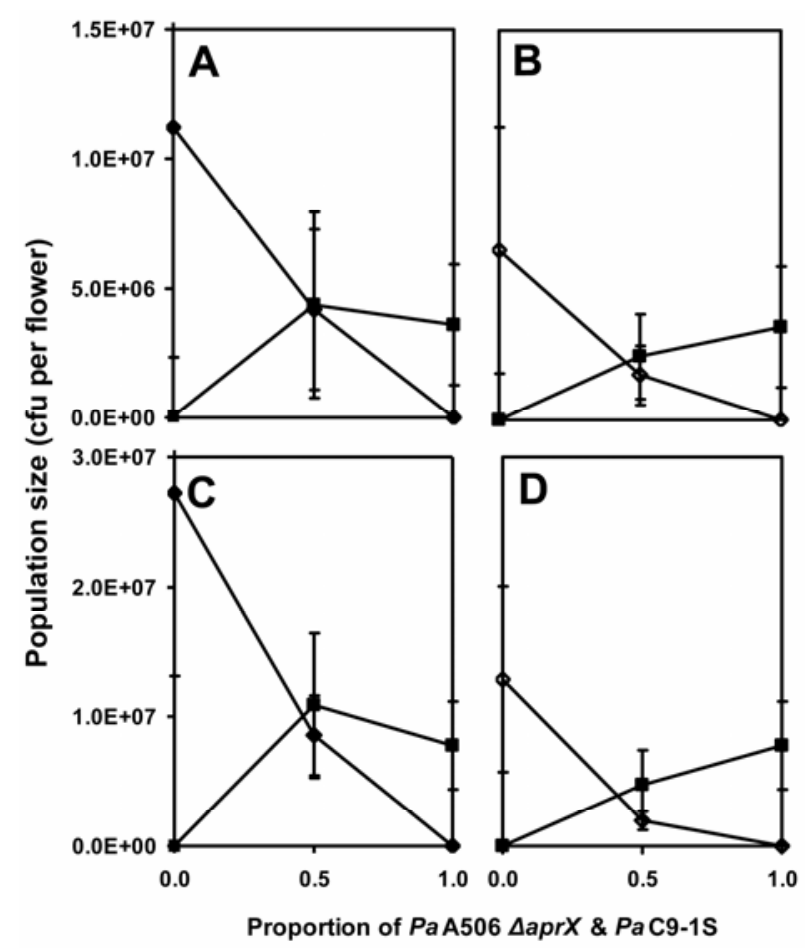

1 - proportion of Ea153N 1 - proportion of Ea153 hrpl-

Fig. 3. Replacement series experiments showing epiphytic population sizes of A and C, virulent Erwinia amylovora strain 153N (solid diamond) and B and D, avirulent E. amylovora strain $153 \mathrm{hrpL}$ (open diamond), each paired against a 1:1 mixture of Pseudomonas fluorescens strain A506 $\Delta a p r X$ and Pantoea agglomerans strain C9-1S (solid square) on pear and apple flowers on screenhouse-grown trees at $96 \mathrm{~h}$ after inoculation. Means represents five experimental replications blocked by inoculation date, two of which were conducted with 'Bartlett' pear, two with 'Golden Delicious' apple, and one 'Snowdrift' crab apple. A and B, Top panels represent data from screenhouse trees grown under ambient conditions; $\mathbf{C}$ and $\mathbf{D}$, bottom panels represent data from trees enclosed in polyethylene film chambers, 1.7 by 1.7 by $2 \mathrm{~m}$ (height), and heated with two 100-W light bulbs during the incubation period. In a replacement series experiment, the proportion of each strain component in the initial inoculum is varied ( $\mathrm{x}$-axis) while keeping the total inoculum concentration constant. Bars represent \pm one standard error of the mean.

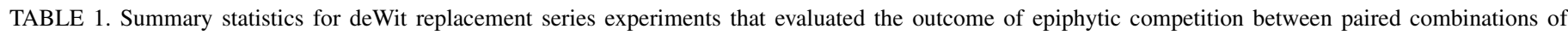

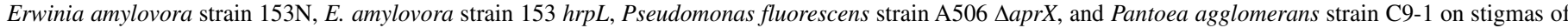
detached pear, apple, and blackberry flowers growing in a growth-chamber environment ${ }^{\mathrm{y}}$

\begin{tabular}{|c|c|c|c|c|c|c|c|c|}
\hline \multirow{2}{*}{\multicolumn{2}{|c|}{ Strain pairing }} & \multirow[b]{3}{*}{ Host flower } & \multicolumn{6}{|c|}{ Outcome of competition $^{\mathrm{z}}$} \\
\hline & & & \multicolumn{3}{|c|}{ First pairing } & \multicolumn{3}{|c|}{ Second pairing } \\
\hline First & Second & & Outcome & $F$ & $P$ value & Outcome & $F$ & $P$ value \\
\hline \multirow[t]{3}{*}{ P. fluorescens A506 $\Delta a p r X$} & P. agglomerans C9-1 & Pear & $\sim$ & 0.6 & n.s. & $\sim$ & 0.4 & n.s. \\
\hline & & Apple & $\sim$ & 1.1 & n.s. & + & 4.4 & 0.0283 \\
\hline & & Blackberry & $\sim$ & 1.5 & n.s. & $\sim$ & 2.9 & n.s. \\
\hline \multirow[t]{3}{*}{ E. amylovora $153 \mathrm{hrpL}$} & A506 $\Delta a p r X / C 9-1 \mathrm{Mix}$ & Pear & - & 9.1 & 0.0020 & $\sim$ & 1.8 & n.s. \\
\hline & & Apple & - & 32.2 & $<0.0001$ & $\sim$ & 2.5 & n.s. \\
\hline & & Blackberry & - & 60.2 & $<0.0001$ & $\sim$ & 1.9 & n.s. \\
\hline \multirow[t]{3}{*}{ E. amylovora $153 \mathrm{~N}$} & A506 $\Delta a p r X / C 9-1 \mathrm{Mix}$ & Pear & - & 43.3 & $<0.0001$ & + & 8.9 & 0.0023 \\
\hline & & Apple & - & 82.9 & $<0.0001$ & + & 11.2 & 0.0008 \\
\hline & & Blackberry & - & 58.3 & $<0.0001$ & + & 11.0 & 0.0002 \\
\hline \multirow[t]{3}{*}{ E. amylovora $153 \mathrm{~N}$} & E. amylovora $153 \mathrm{hrpL}$ & Pear & $\sim$ & 0.5 & n.s. & + & 4.0 & 0.0420 \\
\hline & & Apple & $\sim$ & 0.4 & n.s. & $\sim$ & 2.3 & n.s. \\
\hline & & Blackberry & $\sim$ & 1.4 & n.s. & $\sim$ & 2.6 & n.s. \\
\hline
\end{tabular}

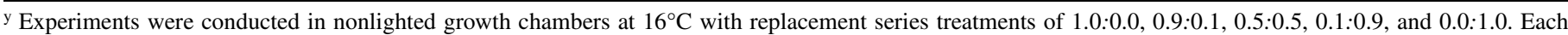
experimental comprised eight detached flowers of Bartlett pear, Gala apple, or Himalayan blackberry inoculated and dilution plated individually; each replacement series was replicated four (pear and apple) or six (blackberry) times.

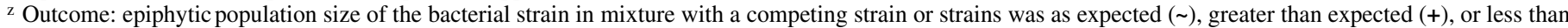
expected (-) relative to the proportion of the strain in the initial inoculum. $F$ statistic and associated $P$ value was based on the analysis of variance model described by Wilson and Lindow (39). Data were blocked by inoculation time; n.s. = not significant. 
Expression of the $d s p E$ promoter by epiphytic $E$. amylovora. Population sizes of virulent gfp constructs of $E$. amylovora on inoculated apple flowers increased from the range of 1 to $2 \times 10^{5}$ $\mathrm{CFU} /$ flower at the time of inoculation to 2 to $3 \times 10^{7} \mathrm{CFU} /$ flower after $96 \mathrm{~h}$ of incubation, with most of the increase occurring over the first $60 \mathrm{~h}$ of incubation (data not shown). As expected, the expression of GFP in bacterial cells from flowers inoculated with Ea153 gfp was 100\%; in contrast and, also as expected, expression of GFP in bacterial cells from flowers inoculated with Ea153 $d s p E^{\text {rev }}:: g f p$ was $0 \%$. Similarly, cells of Ea153 $d s p E:: g f p$ in the initial inoculum suspension and those washed from the flowers sampled immediately after inoculation exhibited no fluorescence indicative of expression of GFP. Cells of Ea153 $d s p E:: g f p$ washed from apple flowers began to show evidence of GFP expression at $24 \mathrm{~h}$ after inoculation (Fig. 5). At this sampling time, $12 \%$ of cells showed green fluorescence, which increased to $29 \%$ of cells at $48 \mathrm{~h}$ after inoculation. Between 48 and $96 \mathrm{~h}$ after inoculation, green fluorescent cells were recovered from flowers at all sampling times, although the proportion of cells showing green fluorescence declined to $<10 \%$ at the end of the period (Fig. 5). In minimal salts medium, each of the constructs attained a maximum population size in the range of $1 \times 10^{7}$ to $1 \times 10^{9} \mathrm{CFU} / \mathrm{ml}$, which occurred between 48 and $72 \mathrm{~h}$ after inoculation; population sizes after $96 \mathrm{~h}$ were generally a $\log$ unit smaller than the observed maxima. Cells of Ea153 dspE::gfp incubated in vials of a minimal salts medium showed a temporal pattern of GFP expression that was similar to cells washed from flowers although, at each sampling time, the proportion of cells from the medium that fluoresced green was typically smaller than observed in the floral washes (Fig. 5).

Fire blight suppression by avirulent $\boldsymbol{E}$. amylovora. Over the nine orchard trials, Ea153 hrpL, PaC9-1S, and PfA506 $\Delta a p r X$, sprayed early to mid-bloom, were readily recovered from flowers sampled from treated trees near petal fall (Table 3). Incidence of recovery for Ea153 hrpL and $\mathrm{PaC} 9-1 \mathrm{~S}$ from treated trees ranged from 76 to $89 \%$ of flowers, with mean populations that generally exceeded $1 \times 10^{5} \mathrm{CFU} /$ flower; PfA506 $\triangle a p r X$ was recovered from $58 \%$ of treated flowers, with mean at populations that averaged $>5 \times 10^{4} \mathrm{CFU} /$ flower. Also, at petal fall, the three sprayed strains were recovered from 10 to $39 \%$ of flowers on trees that were not treated with these bacteria; mean population sizes on flowers from nontreated trees, however, were considerably smaller $(\approx 1.5 \log$ units) than measured on treated trees.

Virulent Ea153N was recovered from high proportions (72 to $87 \%$ ) of flowers sampled at petal fall, with a mean population size on water-treated trees that averaged $\approx 1 \times 10^{5} \mathrm{CFU} /$ flower. On flowers from trees treated with a bacterial antagonists or Ea153 $h r p L$, both incidence of recovery and mean population size of
Ea153N were somewhat smaller than compared with the watertreated control; however, these differences were generally not significant $(P>0.05)$. The lowest incidence of recovery of Ea153N occurred on trees that received the mixture of PaC9-1S and PfA506 $\triangle a p r X$; the lowest mean population size for Ea153N was obtained from flowers on trees treated with Ea153 hrpL (Table 3).

Fire blight symptoms developed in all orchard trials with moderate to high incidences disease (i.e., 66 to 1,621 infected flowers cluster per tree) measured on water-treated trees in all but one of the experiments (Table 4). Compared with the water treat-

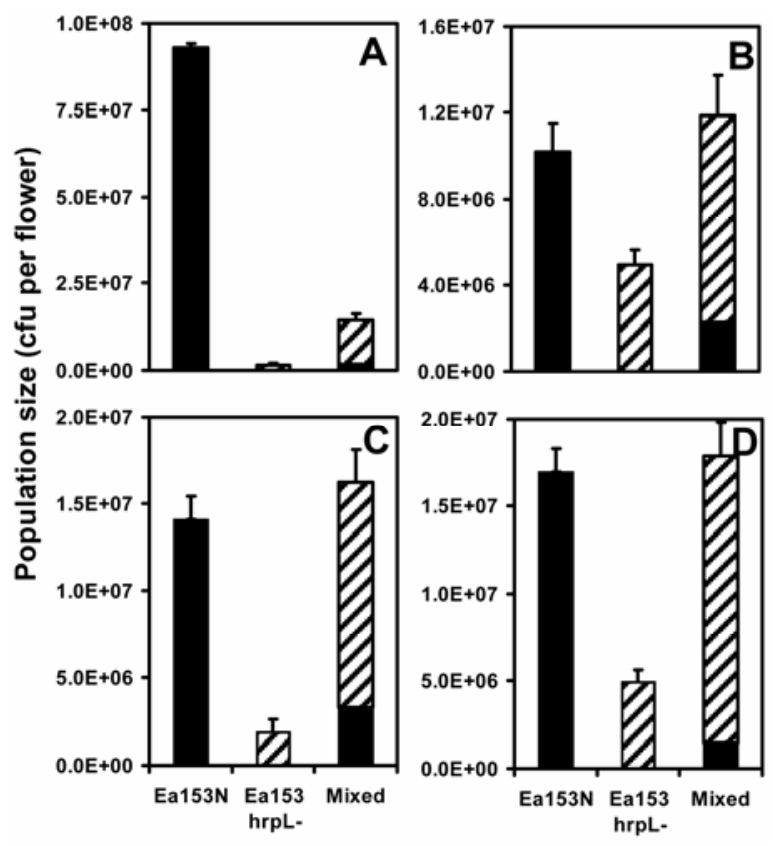

Fig. 4. Epiphytic yield experiments depicting the population sizes of virulent Erwinia amylovora strain 153N (Ea153N; solid bar on left) and avirulent $E$. amylovora strain $153 \mathrm{hrpL}$ (Ea153 hrpL; hatched bar in center) after being inoculated individually and incubated for $96 \mathrm{~h}$ on flowers of A and C, Bartlett pear and $\mathbf{B}$, Gala and $\mathbf{D}$, Golden Delicious apple. Bar on right depicts the total epiphytic yield after inoculation of a mixture of Ea153N (solid portion of bar) and Ea153 hrpL (hatched portion of bar); the initial ratio of virulent (Ea153N) to avirulent (Ea153 $\mathrm{hrpL}$ ) cells in the mixed inoculum ranged from 1:11 to 1:21. A and B, Top panels represent data from detached flowers incubated at $16^{\circ} \mathrm{C}$ in growth chambers; $\mathbf{C}$ and $\mathbf{D}$, lower panels represent data from flowers on screenhouse trees enclosed in polyethylene film chambers, 1.7 by 1.7 by $2 \mathrm{~m}$ (height), and heated with two 100-W light bulbs during the incubation period. Means depict four experimental replications, with 16 flowers representing an experimental unit within a replication; error bars represent \pm one standard error of the mean.

TABLE 2. Summary statistics for deWit replacement series experiments that evaluated the outcome of epiphytic competition between Erwinia amylovora strain $153 \mathrm{~N}$ or E. amylovora strain $153 \mathrm{hrpL}$ paired against a 1:1 mixture of Pseudomonas fluorescens strain A506 $\Delta a p r X$ and Pantoea agglomerans strain C9-1 on stigmas of pear and apple flowers growing in a screenhouse environment ${ }^{y}$

\begin{tabular}{|c|c|c|c|c|c|c|c|c|}
\hline \multirow{2}{*}{\multicolumn{2}{|c|}{ Strain pairing }} & \multirow[b]{3}{*}{ Temperature treatment } & \multicolumn{6}{|c|}{ Outcome of competition ${ }^{\mathrm{z}}$} \\
\hline & & & \multicolumn{3}{|c|}{ First pairing } & \multicolumn{3}{|c|}{ Second pairing } \\
\hline First & Second & & Outcome & $F$ & $P$ value & Outcome & $F$ & $P$ value \\
\hline \multirow{2}{*}{ E. amylovora $153 \mathrm{hrpL}$} & & Chambered & - & 10.1 & 0.0041 & $\sim$ & 0.2 & n.s. \\
\hline & A506 $\Delta a p r X / C 9-1 \mathrm{Mix}$ & Ambient & $\sim$ & 0.4 & n.s. & + & 5.0 & 0.035 \\
\hline E. amylovora $153 \mathrm{~N}$ & & Chambered & $\sim$ & 3.0 & n.s. & + & 8.9 & 0.0064 \\
\hline
\end{tabular}

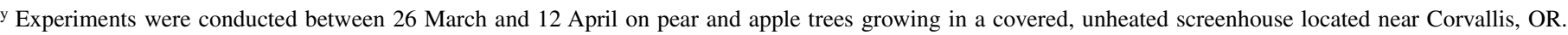
For each pairing, replacement series treatments of 1.0:0.0, 0.5:0.5, and 0.0:1.0 were inoculated twice on pear cv. Bartlett and apple cv. Golden delicious and once on crab apple cv. Snowdrift. At each inoculation time, bacterial suspensions $\left(1 \times 10^{7} \mathrm{CFU} / \mathrm{ml}\right)$ representing all replacement series treatments were misted onto newly open flowers on marked branches of each of six trees. A temperature treatment was created by enclosing half the trees in 1.7-by-1.7-by-2-m polyethylene film chambers and heating the chambers with a 100-watt light bulb during a 96-h incubation period. Each experiment consisted of four flowers which were sampled and dilution plated individually; sampling times were at 1 and $96 \mathrm{~h}$ after inoculation.

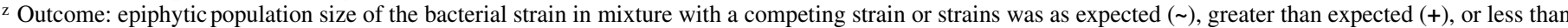
expected (-) relative to the proportion of the strain in the initial inoculum. $F$ statistic and associated $P$ value was based on the analysis of variance model described by Wilson and Lindow (39). Data were blocked by inoculation time; n.s. = not significant. 
ment, biological treatments (Ea153 hrpL and the mixture of PaC9-1S and PfA506 $\triangle a p r X$, with or without Ea153 hrpL) reduced the incidence of fire blight by an average of 37 to $44 \%$, which was similar to the $44 \%$ reduction achieved with two applications of oxytetracycline but less than the $79 \%$ reduction achieved by streptomycin sulfate. The mixture of PaC9-1S and PfA506 $\triangle a p r X$ significantly reduced disease incidence in six of nine trials but Ea153 hrpL provided significant control of fire blight in only four of the trials; Ea153 hrpL combined with PaC9-1S and PfA506 $\Delta a p r X$ provided significant fire blight control in five of the nine experiments.

\section{DISCUSSION}

Understanding competitive interactions among antagonists and a target pathogen is a key to making optimal use of biological controls. Replacement series experiments, a formal design originally introduced by de Wit (8) as a tool for studying competition between plant species, have been insightful for understanding

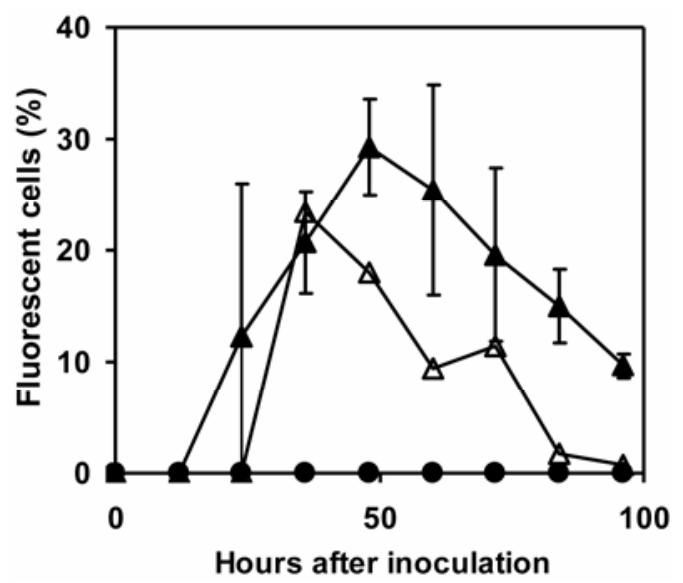

Fig. 5. Expression in green fluorescent protein in Erwinia amylovora construct Ea153 dspE::gfp (A) and control construct Ea153 $d s p E^{\mathrm{rev}}: \because g f p(\mathbf{O})$ growing epiphytically on stigmas of detached apple flowers, and in Ea153 $d s p E:: g f p$ cultured in a minimal salts medium broth $(\Delta)$ in relation to hours after inoculation. The experiment also included the control construct Ea153 $g f p$, for which $100 \%$ of the cells expressed green fluorescent protein (GFP) (data not shown). The incidence of fluorescence was determined by microscopic examination of a 100-cell subsample from each of six (E. amylovora $153 d s p E:: g f p$ ) or three (other constructs) flowers within each experimental replication. Means shown represent four experimental replications, with vertical bars drawn through points of E. amylovora 153 dspE::gfp depicting \pm one standard deviation of the mean. interactions among phyllosphere bacteria (40). In this kind of experiment, the initial inocula of competing bacterial strains are mixed in varying proportions but at a constant total density. After an incubation period, the resultant population sizes (i.e., epiphytic yields) of each strain in the mixtures are compared with the result when each species is grown alone. Interpretation of replacement series experiments is based on comparison of results to several hypothesized outcomes, including antagonism, mutualism, and commensalism (8). For our study, consistent results from replacement series inoculations of floral stigmas on several hosts and in different environments provided several insights and conclusions. First, and not as a result of direct competition, the final population size and, therefore, the growth rate of populations of virulent Ea153N was always significantly higher than that of populations of avirulent Ea153 hrpL or the mixture of nonpathogenic bacterial antagonists, even on the nondisease host Himalayan blackberry (Fig. 2G and H). This is a result we have observed previously (17) and, in part, our earlier observations of a high growth rate by virulent $E$. amylovora on floral stigmas of both disease hosts $(14,17)$ and rosaceous, nondisease hosts $(14)$ served as a rationale for this investigation. Second, and not unexpectedly $(16,26,29)$, growth of both virulent and avirulent strains of E. amylovora on floral stigmas was antagonized by the mixture of PaC9-1S and PfA506 $\Delta a p r X$. That is, the measured final epiphytic yield of pathogenic and avirulent E. amylovora was apparently less than the expectation of linearity with initial inoculum proportion (Fig. $2)$; this result was statistically significant for all growth-chamber experiments (Table 1) but, in the screenhouse experiment, it was significant only for Ea153 hrpL on pear and apple flowers maintained in the chambered environment (Table 2). Third, and surprisingly, in both the growth-chamber and screenhouse environments, the final population sizes of the antagonist strains in mixture with virulent Ea153N were greater than the expectation of linearity with initial inoculum proportion but this was not true for the interaction of the antagonist mixture with the avirulent hrpL mutant of Ea153 (Tables 1 and 2). Thus, the antagonists appeared to benefit more from the interaction with virulent Ea153N than they did from interactions with the avirulent mutant. In the growth chamber, avirulent Ea153 hrpL also appeared to benefit from the interaction with its virulent parent (note particularly the inoculum ratios of 0.1:0.9 Ea153N to Ea153 hrpL) (Fig. 2) but this result was statistically significant only in the experiment with pear (Table 1).

The competitive interaction of Ea153N and Ea153 hrpL when the initial composition of the mixed inoculum was skewed toward a high proportion of the avirulent strain was investigated in additional experiments. The epiphytic yield of solitary inoculations of each strain again showed that the virulent strain attained

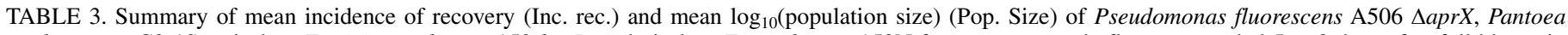

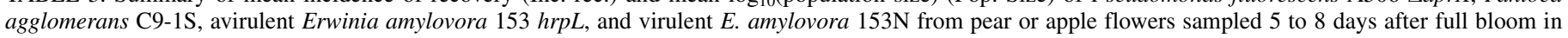
nine experiments to which biological agents or water were applied to orchards located near Corvallis, OR during 2004 to $2007^{\mathrm{x}}$

\begin{tabular}{|c|c|c|c|c|c|c|c|c|}
\hline \multirow[b]{3}{*}{ Treatment $^{\mathrm{y}}$} & \multicolumn{8}{|c|}{ Bacterial strain ${ }^{z}$} \\
\hline & \multicolumn{2}{|c|}{ Pseudomonas fluorescens } & \multicolumn{2}{|c|}{ Pantoea agglomerans } & \multicolumn{2}{|c|}{ E. amylovora hrpL } & \multicolumn{2}{|c|}{ E. amylovora $153 \mathrm{~N}$} \\
\hline & Inc. rec. & Pop. size & Inc. rec. & Pop. size & Inc. rec. & Pop. size & Inc. rec. & Pop. size \\
\hline Water & $0.10(0.09)$ & $3.50(0.82)$ & $0.38(0.29)$ & $3.90(0.74)$ & $0.25(0.25)$ & $3.17(1.32)$ & $\mathbf{0 . 8 6}(0.10)$ & $5.08(1.13)$ \\
\hline Ea153 hrpL & $0.10(0.13)$ & $3.08(1.33)$ & $0.39(0.32)$ & $3.60(0.98)$ & $\mathbf{0 . 9 0}(0.11)$ & $5.29(0.65)$ & $0.76(0.16)$ & $4.63(1.02)$ \\
\hline PfA506 $\triangle a p r X$ and PaC9-1S & $\mathbf{0 . 6 4}(0.22)$ & $4.54(0.72)$ & $0.84(0.15)$ & $5.28(0.70)$ & $0.19(0.18)$ & $3.80(1.18)$ & $\mathbf{0 . 6 7}(0.19)$ & $4.59(0.80)$ \\
\hline PfA506 $\Delta a p r X$, PaC9-1S, and Ea153 hrpL & $0.64(0.25)$ & $4.70(0.62)$ & $0.85(0.19)$ & $5.18(0.75)$ & $0.76(0.22)$ & $4.66(0.62)$ & $\mathbf{0 . 7 0}(0.21)$ & $4.88(0.72)$ \\
\hline
\end{tabular}

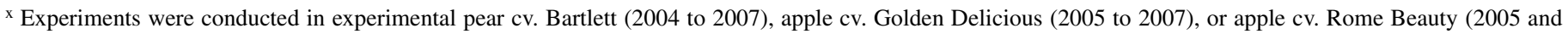
2006) orchards located near Corvallis, OR.

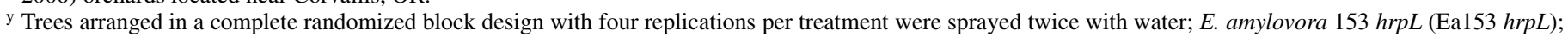
a 1:1 mix of Pseudomonas fluorescens A506 $\Delta a p r X$ (PfA506 $\Delta a p r X)$ and Pantoea agglomerans C9-1 (PaC9-1); and a 1:1:1 mix of PfA506 $\Delta a p r X$, PaC9-1, and Ea153 $\mathrm{hrpL}$ at 30 and $70 \%$ bloom. At full bloom, the pathogen Ea153N was applied at $5 \times 10^{5}$ to $1 \times 10^{6} \mathrm{CFU} / \mathrm{ml}$.

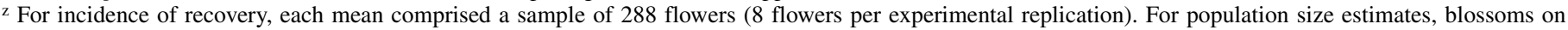
which a bacterial strain was not detected were excluded from the mean. Stain means presented in bold correspond to treatments sprayed with that strain. Numbers in parentheses are standard deviation among means of individual experiments. 
a higher population size than the avirulent strain on detached flowers in growth chambers and on flowers on trees growing in a screenhouse. More interestingly, the avirulent Ea153 hrpL significantly benefited from the interaction with the virulent parent as shown by the enhanced final population sizes of avirulent Ea153 $h r p L$ after the addition of a small proportion (4 to 11\%) of virulent cells to the initial inoculum (Fig. 4). Nonetheless, in spite of enhanced yields of the avirulent strain when grown in combination with the virulent strain, over the incubation period, the virulent strain approximately doubled its proportion in the total population (i.e., from 4 to $11 \%$ to 8 to $20 \%$ ) indicating a higher growth rate and, likely, a unique ability within the stigmatic niche compared with an nearly isogenic strain that lacked the ability to cause disease.

Because virulent E. amylovora shows larger epiphytic yields and because it apparently enhances the epiphytic yields of nonpathogenic co-inhabitants, we have developed the hypothesis that virulent E. amylovora uses its ability to cause disease to expand or modify the epiphytic habitat $(20,35,39)$, thereby providing itself a competitive advantage over nonpathogens. Importantly, pathogenesis-related activity by E. amylovora within the secretory, nutrient-rich environment of the stigma increases the carrying capacity of the habitat, which allows nonpathogenic coinhabitants to grow to a greater-than-expected population size. We speculate that niche modification involves microsites on and within the stigma where E. amylovora can associate intimately or, perhaps, progress intracellularly. Moreover, these modified sites may be less available to nonpathogens (39). An example of such pathogen-formed sites may be shown in the confocal image from Spinelli et al. (25; Fig. 4B from reference 25), which shows cocolonization of apple stigmas by a red fluorescent protein-labeled E. amylovora and by green fluorescent protein-labeled Pantoea agglomerans strain C9-1. In this image, a layer of $P$. agglomerans strain C9-1 is shown occupying the outer (most distal) areas of the stigmatic surface but small, concentrated aggregates of redlabeled E. amylovora are apparent near the bases of the secretory, columnar papillae that compose the stigmatic surface. Similarly, Faize et al. (5) reported that, after inoculation of apple seedlings with a mixture of a virulent and an avirulent $h r p L$ mutant of $E$. amylovora, the two strains localized in separate areas of leaf tissue.

Important to our hypothesis is that the type III secretion system (TTSS) of E. amylovora is functional and hrp genes are expressed when the pathogen is growing as an epiphyte on a host stigma. Based on gene-expression experiments in culture media, it is frequently noted that the TTSS of bacterial plant pathogens are not expressed in nutrient-rich environments $(30,37)$. Moreover, within the specific etiology of fire blight, although the nutrientrich environment of the stigma supports large epiphytic populations of the pathogen, it is not the site of systemic floral infection; instead, pathogen cells migrate to the base of the style and enter the plant through openings (nectarthodes) on the nectary. Consequently, we evaluated the regulation of expression of a pathogenesis-related gene, $d s p E$, with a $g f p$ reporter system specifically to provide evidence of pathogenesis-related activity in a nutrientrich environment distal from the site of infection (i.e., the stigmatic surface). DspE, which is analogous to AvrE of Pseudomonas syringae and whose production is regulated by $h r p L$, is considered a critical, secreted effector protein in the diseaseinduction process $(2,3,42)$. Our result showing a sizable proportion of the epiphytic cells of Ea153 $d s p E:: g f p$ expressing GFP at 24 to $96 \mathrm{~h}$ after inoculation indicates a potential for pathogenesisrelated activity to occur on or within the stigmatic tissues. Although this evidence is only indirect, these data suggest that $E$. amylovora is capable of pathogenesis-related activity when growing as an epiphyte on stigmas, and that this activity may provide a virulent strain a competitive advantage over a nonpathogen when competing as an epiphyte on flowers.

The results of field trials using Ea153 hrpL as a biocontrol agent were consistent with our introductory remarks concerning the partial effectiveness of biological controls for fire blight, and also were consistent with the conclusion from the competition experiments that virulent strains of the pathogen show superior fitness to avirulent strains (and to nonpathogens) as epiphytes on pear and apple flowers. The hrpL mutant of Ea153 was an excellent colonist of stigmatic surfaces, and significantly suppressed the incidence of fire blight in several trials. Nonetheless, inoculations of virulent E. amylovora, delayed in time and reduced in dose relative to the biocontrol treatments, still caused significant disease on trees treated with the avirulent mutant of Ea153. Also, the performance of the hrpL mutant of Ea153 as a biocontrol agent was slightly inferior to that of the antagonist mixture (PaC9-1S and PfA506 $\Delta a p r X$ ), perhaps because the mixture utilizes the mechanism of antibiosis (29) whereas the avirulent strain of the pathogen does not. Induction of host defenses has been proposed as a mechanism by which hrp regulatory mutants

TABLE 4. Relative incidence of fire blight on pear and apple trees treated with water, Pseudomonas fluorescens A506 DaprX, Pantoea agglomerans C9-1S and Erwinia amylovora $153 \mathrm{hrpL}$ or commercial antibiotics in experimental orchards located in Corvallis, OR during 2004 to 2007

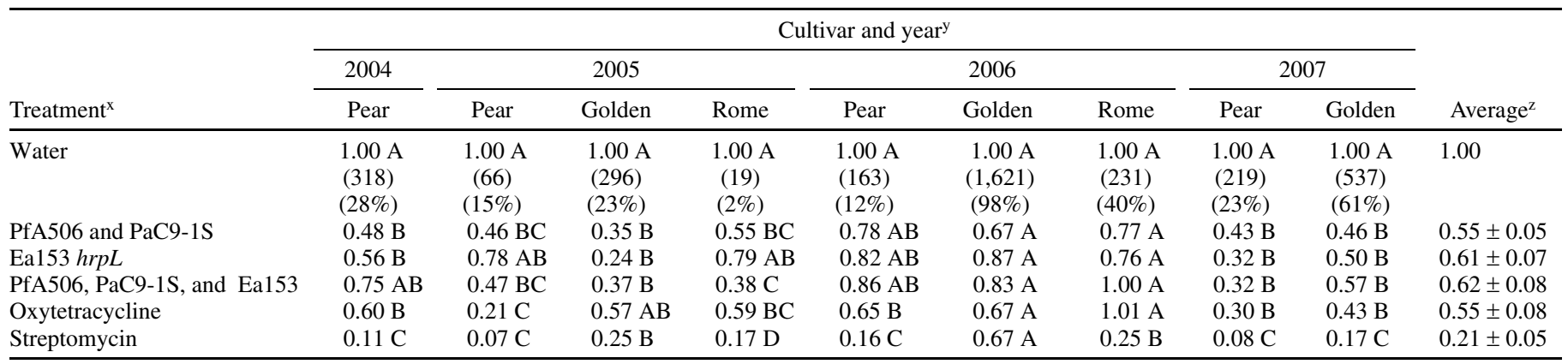

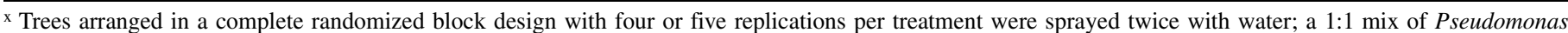
fluorescens A506 DaprX (PfA506) and Pantoea agglomerans C9-1 (PaC9-1); E. amylovora 153 hrpL (Ea153); and a 1:1:1 mix of PfA506, PaC9-1, and Ea153 hrpL at 30 and $70 \%$ bloom. At full bloom, the pathogen Ea153N was applied at $5 \times 10^{5}$ to $1 \times 10^{6} \mathrm{CFU} / \mathrm{ml}$. The antibiotic standards oxytetracycline and streptomycin were applied at $70 \%$ bloom and repeated within $36 \mathrm{~h}$ after inoculation with the streptomycin- and oxytetracycline-sensitive pathogen $E$. amylovora $153 \mathrm{~N}$.

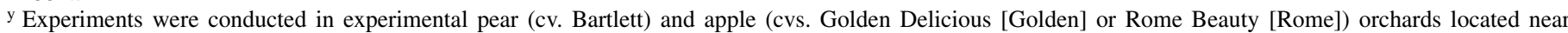
Corvallis, OR. Different letters within a column following the incidence of fire blight relative to water-treated trees indicate a significant difference in the arcsine square root transformed disease incidence data by Fischer's protected least significance difference at $P=0.05$. Top number in parentheses is the average number of fire blight strikes on water-treated trees. The bottom number in parentheses is mean percentage of blossom clusters blighted on water-treated trees; relative disease incidence was determined for each treatment by dividing through with this percentage.

$\mathrm{z}$ Treatment average \pm standard error of the mean. 
of E. amylovora protect apple seedlings from ingress and internal spread of the pathogen (5). The hypothesis that hrp mutants induce host defenses also was proposed as a result of field trials for biocontrol of bacterial spot of tomato where the amount of disease suppression obtained by weekly treatment with avirulent derivatives of Xanthomonas campestris pv. vesicatoria depended greatly on the specific hrp mutation (23).

In conclusion, the ability to cause disease via a functional TTSS is apparently the reason that E. amylovora shows superior fitness as an epiphyte on the stigmas of rosaceous plants relative to bacteria that do not possess this ability. Interestingly, growth of virulent $E$. amylovora on stigmatic tissues enhances the epiphytic yields of nonpathogenic cohabitants, perhaps through the release of additional nutrients or by suppression of defense responses owing to pathogenesis-related activities of the virulent strain. The implication for biological control of fire blight is that, as an epiphyte, virulent $E$. amylovora has special abilities relative to antagonists applied to flowers to suppress epiphytic growth of the pathogen. This may explain why it has been difficult to select antagonists that can achieve more than the typical 40 to $60 \%$ suppression of floral infection in pathogen-inoculated field trails. In addition, with the principle site of epiphytic growth physically distinct from the site of systemic infection, it also shows the uniqueness of fire blight as a model for further study of pathogenesis-related activity contributing to epiphytic fitness of bacterial pathogens.

\section{ACKNOWLEDGMENTS}

Research support was provided by the United States Department of Agriculture (USDA) National Research Initiative Competitive Grant No. 2003-03374 and by the Oregon State University Agricultural Experiment Station. The avirulent $h r p L$ mutant of E. amylovora strain Ea153 was field released under USDA APHIS permit no. 03-279-01r.

\section{LITERATURE CITED}

1. Anderson, L. M., Stockwell, V. O., and Loper, J. E. 2004. An extracellular protease of Pseudomonas fluorescens inactivates antibiotics of Pantoea agglomerans. Phytopathology 94:1228-1234.

2. Bogdanove, A. J., Kim, J. F., and Beer, S. V. 2000. Disease specific genes of Erwinia amylovora: Keys to understanding pathogenesis and potential targets for control. Pages 163-178 in: Fire Blight: The Disease and Its Causative Agent, Erwinia amylovora. J. L. Vanneste, ed. CAB International, London.

3. Boureau, T., El Maarouf-Bouteau, H., Garnier, A., Brisset, M.-N., Perino, C., Pucheu, I., and Barny, M. A. 2006. DspA/E, a type III effector essential for Erwinia amylovora pathogenicity and growth in planta, induces cell death in host apple and nonhost tobacco plants. Mol. PlantMicrobe Interact. 9:16-24.

4. Chang, J. H., Urbach, J. M., Law, T. F., Arnold, L. W., Hu, A., Gombar, S., Grant, S. R., Ausubel, F. M., and Dangl, J. L. 2005. A highthroughput, near-saturating screen for type III effector genes from Pseudomonas syringae. Proc. Natl. Acad. Sci. U.S.A. 102:2549-2554.

5. Faize, M., Brisset, M. N., Perino, C., Vian, B., Barny, M. A., Paulin, J. P., and Thauraud, M. 2006. Protection of apple against fire blight induced by an hrpL mutant of Erwinia amylovora. Biol. Plant. 50:667-674.

6. Frey, P., Prior, P., Marie, C., Kotoujansky, A., Trigalet-Demery, D., and Trigalet, A. 1994. Hrp ${ }^{-}$mutants of Pseudomonas solanacearum as potential biocontrol agents of tomato bacterial wilt. Appl. Environ. Microbiol. 60:3175-3181.

7. Goodman, R. N. 1966. Protection of apple stem tissue against Erwinia amylovora infection by avirulent strains and three other bacterial species. Phytopathology 57:22-24.

8. Harper, J. L. 1977. Population Biology of Plants. Academic Press, London.

9. Hirano, S. S., Charkowski, A. O., Collmer, A., Willis, D. K. and Upper, C. D. 1999. Role of the Hrp type III protein secretion system in growth of Pseudomonas syringae pv. syringae B728a on host plants in the field. Proc. Natl. Acad. Sci. U.S.A. 96:9851-9856.

10. Hirano, S. S., Nordheim, E. V., Arny, D. C., and Upper, C. D. 1982. Lognormal distribution of epiphytic bacteria populations on leaf surfaces. Appl. Environ. Microbiol. 44:695-700.

11. Huynh, T. V., Dahlbeck, D., and Staskawicz, B. J. 1989. Bacterial blight of soybean: Regulation of a pathogen gene determining host cultivar specificity. Science 245:1374-1377.

12. Ishimaru, C. A., and Klos, E. J. 1984. New medium for detecting Erwinia amylovora and its use in epidemiological studies. Phytopathology 74:1342-1345.

13. Ishimaru, C. A., Klos, E. J., and Brubaker, R. R. 1988. Multiple antibiotic production by Erwinia herbicola. Phytopathology 78:746-750.

14. Johnson, K. B., Sawyer, T. L., and Temple, T. N. 2006. Rates of epiphytic growth of Erwinia amylovora on flowers common in the landscape. Plant Dis. 90:1331-1336.

15. Johnson, K. B., and Stockwell, V. O. 1998. Management of fire blight: A case study in microbial ecology. Annu. Rev. Phytopathol. 36:227-248.

16. Johnson, K. B., Stockwell, V. O., McLaughlin, M. J., Sugar, D., Loper, J. E., and Roberts, R. G. 1993. Effect of bacterial antagonists on establishment of honey bee-dispersed Erwinia amylovora in pear blossoms and on fire blight control. Phytopathology 83:995-1002.

17. Johnson, K. B., Stockwell, V. O., and Sawyer, T. L. 2004. Adaptation of fire blight forecasting to optimize the use of biological controls. Plant Dis. $88: 41-48$.

18. Lindow, S. E., McGourty, G., and Elkins, R. 1996. Interactions of antibiotics with Pseudomonas fluorescens A506 in the control of fire blight and frost injury of pear. Phytopathology 86:841-848.

19. Loper, J. E., Suslow, T. V., and Schroth, M. N. 1984. Lognormal distribution of bacterial populations in the rhizosphere. Phytopathology 74:1454-1460

20. Marco, M. L., Legac, J., and Lindow, S. E. 2005. Pseudomonas syringae genes induced during colonization of leaf surfaces. Environ. Microbiol. 7:1379-1391.

21. Metzger, M., Bellemann, P., Schwartz, T., and Geider, K. 1992. Sitedirected and transposon-mediated mutagenesis with pfd-plasmids by electroporation of Erwinia amylovora and Escherichia coli cells. Nucleic Acids Res. 20:2265-2270.

22. Miller, T. D., and Schroth, M. N. 1972. Monitoring the epiphytic population of Erwinia amylovora on pear with a selective medium. Phytopathology 62:1175-1182.

23. Moss, W. P., Byrne, J. M., Campbell, H. L, Ji, P., Bonas, U., Jones, J. B., and Wilson, M. 2007. Biological control of bacterial spot of tomato using hrp mutants of Xanthomonas campestris pv. vesicatoria. Biol. Control 41:199-206.

24. Pusey, P. L. 1997. Crab apple blossoms as a model for research on biological control of fire blight. Phytopathology 87:1096-1102.

25. Spinelli, F., Ciampolini, F., Cresti, M., Geider, K., and Costa, G. 2005. Influence of stigmatic morphology on flower colonization by Erwinia amylovora and Pantoea agglomerans. Eur. J. Plant Pathol. 113:395-405.

26. Stockwell, V. O., Anderson, L. M., Johnson, K. B., and Loper, J. E. 2001. Suppression of fire blight with mixtures of mechanistically-compatible biological control agents. (Abstr.) Phytopathology 91:S190.

27. Stockwell, V. O., Johnson, K. B., and Loper, J. E. 1998. Establishment of bacterial antagonists of Erwinia amylovora on pear and apple blossoms as influenced by inoculum preparation. Phytopathology 88:506-513.

28. Stockwell, V. O., Johnson, K. B., and Loper, J. E. 2001. Biological control of fire blight: Understanding interactions among introduced and indigenous microbial communities. Pages 225-240 in: Phylllosphere Microbiology. S. E. Lindow, E. I. Hecht-Poinar, and V. J. Elliott, eds. American Phytopathological Society Press, St. Paul, MN.

29. Stockwell, V. O., Johnson, K. B, Sugar, D., and Loper, J. E. 2002. Antibiosis contributes to biological control of fire blight by Pantoea agglomerans strain Eh252 in the field. Phytopathology 92:1202-1209.

30. Tang, X., Xiao, Y., and Zhou, J.-M. 2006. Regulation of the type III secretion system in phytopathogenic bacteria. Mol. Plant-Microbe Interact. 19:1159-1166.

31. Temple, T. N., Stockwell, V. O., Pusey, P. L., and Johnson, K. B. 2007. Evaluation of likelihood of co-occurrence of Erwinia amylovora with mature fruit of winter pear. Phytopathology 97:1263-1273.

32. Tharaud, M., Laurent, J., Faize, M., and Paulin, J. P. 1997. Fire blight protection with avirulent mutants of Erwinia amylovora. Microbiology 143:625-632.

33. Thomson, S. V. 1986. The role of the stigma in fire blight infections. Phytopathology 76:476-482.

34. Van der Zwet, T., and Beer, S. V. 1992. Fire Blight—its nature, prevention, and control: A practical guide to disease management. Agric. Inf. Bull. 613. USDA Science and Education Administration, Washington, DC.

35. Vinatzer, B. A., Teitzel, G. M., Lee, M. W., Jelenska, J., Hotton, S., Fairfax, K., Jenrette, J., and Greenberg, J. T. 2006. The type III effector repertoire of Pseudomonas syringae pv. syringae B728a and its role in survival and disease on host and non-host plants. Mol. Microbiol. 62:2644

36. Wei, Z.-M., and Beer, S. V. 1995. HrpL activates Erwinia amylovora hrp gene transcription and is a member of the ECF subfamily of $\sigma$-factors. J. Bacteriol. 177:6201-6210. 
37. Wei, Z.-M., Kim, J. F., and Beer, S. V. 2000. Regulation of hrp genes and type III protein secretion in Erwinia amylovora by HrpX/HrpY, a novel two-component system, and HrpS. Mol. Plant-Microbe Interact. 13:12511262.

38. Wichmann, G., and Bergelson, J. 2004. Effector genes of Xanthomonas axonopodis pv. vesicatoria promote transmission and enhance other fitness traits in the field. Genetics 166:693-706.

39. Wilson, M., Hirano, S. S., and Lindow, S. E. 1999. Location and survival of leaf-associated bacteria in relation to pathogenicity and potential for growth within the leaf. Appl. Environ. Microbiol. 65:1435-1443.
40. Wilson, M., and Lindow, S. E. 1994. Ecological similarity and coexistence of epiphytic ice-nucleating (Ice+) Pseudomonas syringae strains and a non-ice-nucleating (Ice-) biological control agent. Appl. Environ. Microbiol. 60:3128-3137.

41. Wrather, J. A., Kuc, J., and Williams, E. B. 1973. Protection of apple and pear fruit tissue against fire blight with nonpathogenic bacteria. Phytopathol. Notes 63:1075-1076.

42. Zhao, Y., Blumer, S. E., and Sundin, G. W. 2005. Identification of Erwinia amylovora genes induced during infection of immature pear tissue. J. Bacteriol. 187:8088-8103. 